\title{
Cardiac assistance from skeletal muscle: a critical appraisal of the various approaches
}

\author{
Stanley Salmons, Jonathan C Jarvis
}

British Heart

Foundation Skeletal Muscle Assist Research Group, Department of Human Anatomy and Cell Biology, University of Liverpool, Liverpool S Salmons J C Jarvis

Correspondence to Professor $\mathbf{S}$ Salmons, British Heart Foundation Skeletal Muscle Assist Research Group, Department of Human Anatomy and Cell Biology PO Box 147, University of Liverpool, Liverpool L69 3BX.

Accepted for publication 1 May 1992

\begin{abstract}
We review here various ways in which cardiac assistance might be derived from a patient's own skeletal muscle. Calculations based on experimental data and optimistic estimates of the efficiency of the energy conversions involved suggest that the continuous assist available would be limited to about 2 litres a minute if a muscle were used to energise an electromechanical device. It would be more efficient to couple the energy mechanically or hydraulically, but these approaches still pose problems of anatomical placement, muscle attachment, fluid leakage, and cost. Unless these issues can be addressed, the use of skeletal muscle as an internal power source for mechanical circulatory assist devices will remain an unworkable concept. Configurations that couple skeletal muscle contraction directly to the circulation would be more efficient and less costly. In terms of the energy available, a skeletal muscle ventricle could be designed to provide a continuous partial assist of $1-21 / \mathrm{min}$, with flows of up to $81 / \mathrm{min}$ sustainable for limited periods. Such an approach offers new possibilities for the surgical treatment of chronic cardiac failure.
\end{abstract}

(Br Heart J 1992;68:333-8)

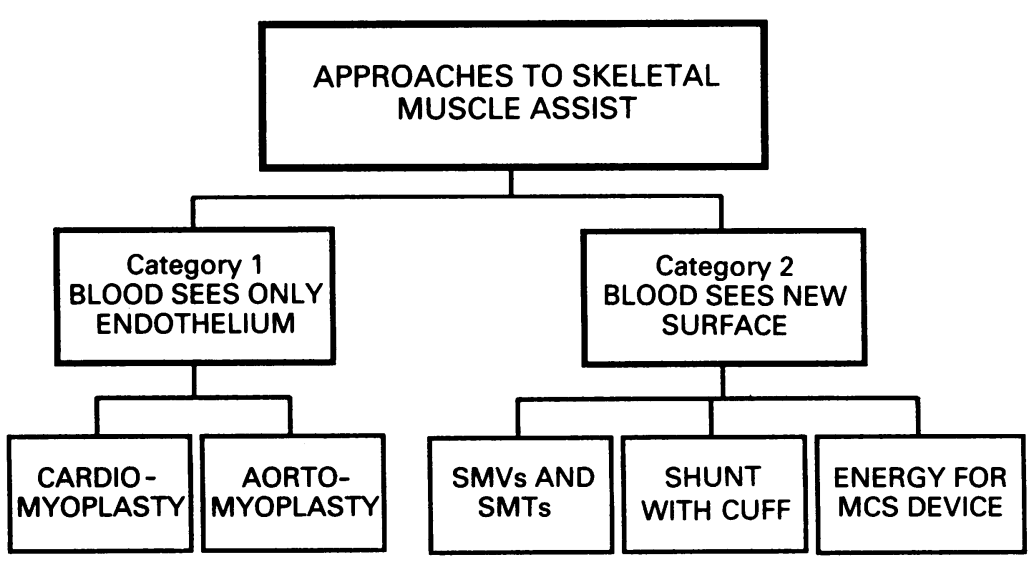

Figure 1 Alternative approaches to the use of skeletal muscle for cardiac assistance. MCS, mechanical circulatory support; SMV, skeletal muscle ventricle; SMT, skeletal muscle tube ventricle.
For some years the surgical treatment of end stage cardiac failure has been restricted to cardiac transplantation and the temporary use of mechanical circulatory support devices as a bridge to transplant. The discovery that adult fast-twitch mammalian skeletal muscle is capable of undergoing adaptive change, through which it can acquire a markedly increased resistance to fatigue, has revived interest in the possibility of diverting skeletal muscle from its normal function to perform in a cardiac assist role.

The adaptive transformation of skeletal muscle that takes place in response to increased use has been studied extensively ${ }^{1-3}$ and it is not proposed to describe it in any detail here. The important elements can, however, be stated quite simply. A muscle will be resistant to fatigue if it can supply, on a continuous basis, sufficient ATP to meet the prevailing energy costs of contraction. These costs derive mainly from the cyclic turnover of chemical bonds between actin and myosin and the transport of calcium between intracellular compartments. Skeletal muscle that has been conditioned by long-term electrical stimulation acquires more favourable bioenergetics for sustained contraction as a result of changes both in the isoforms of myosin and in the kinetics of the release and uptake of calcium. At the same time, sustained production of ATP becomes possible through an increase in the capacity of oxidative pathways, particularly those involved in the breakdown of fat and fatty acids, at the expense of anaerobic glycolysis. There is an associated increase in capillary blood supply and mitochondrial volume. As a result, the production of ATP can match even the extreme increases in the utilisation of ATP required for the performance of cardiac work. ${ }^{4-6}$

Four approaches to skeletal muscle assist There are several ways in which we can try to harness the power of conditioned skeletal muscle for cardiac assistance but they fall into two major categories (fig 1).

\section{CATEGORY 1 ASSISTS}

These preserve the natural endothelial lining of the patient's cardiovascular system. There are two main approaches:

(a) In cardiomyoplasty, the object is to 
Figure 2 Highly schematic examples to illustrate the approaches listed in fig 1 .

(A) cardiomyoplasty,

(B) aortomyoplasty,

(C) compression of aorta by a cuff, (D) skeletal muscle ventricle,

(E) skeletal muscle tube ventricle, $(F)$ left ventricular assist device (LVAD) energised mechanically, (G) LVAD energised hydraulically, (H) LVAD energised electrically.
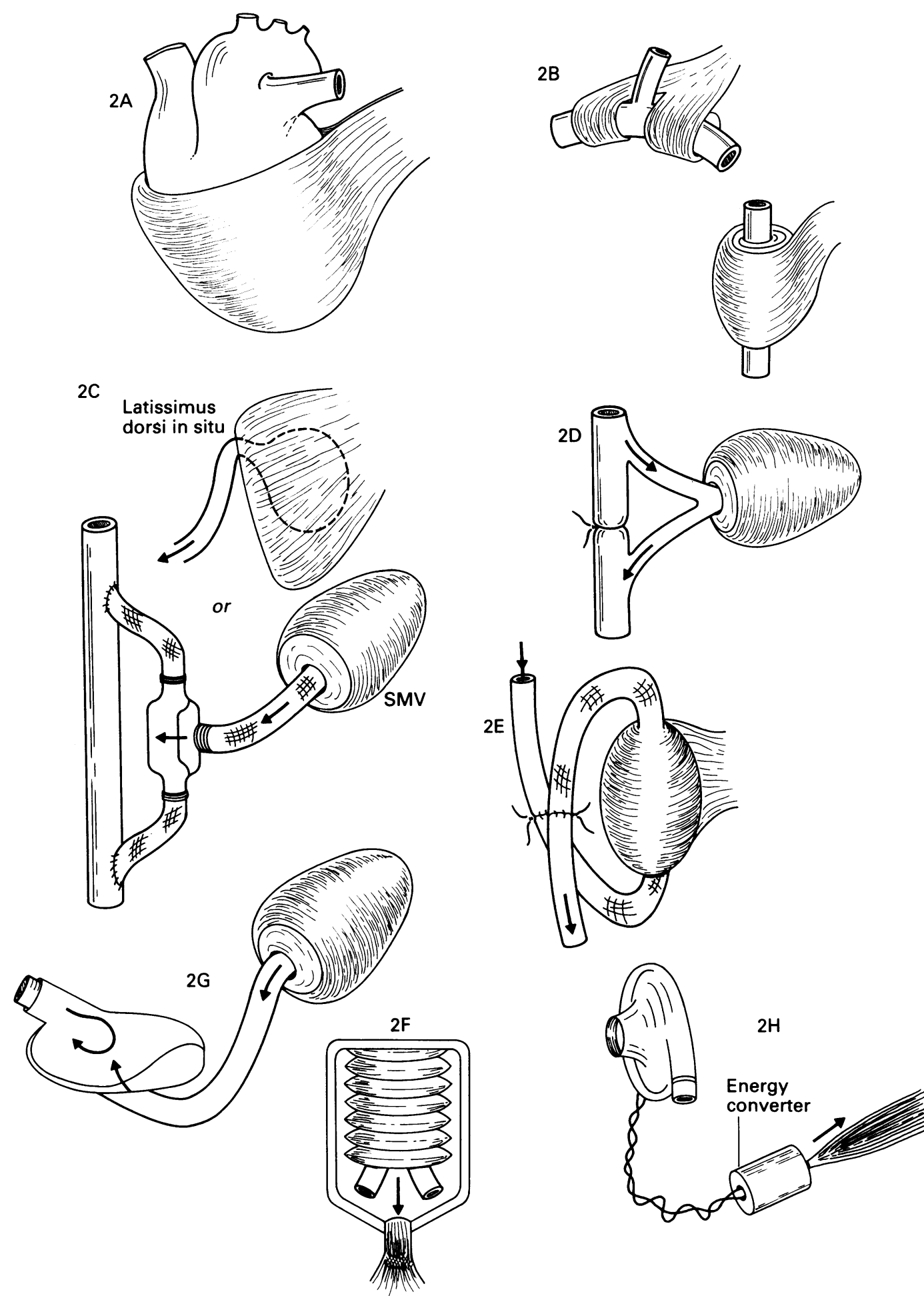

produce intermittent compression of the patient's left or right ventricle by the contraction of skeletal muscle wrapped around the $\operatorname{organ}^{78}$ (fig 2A).

(b) In aortomyoplasty, the ascending or descending aorta may be wrapped directly ${ }^{9}$ (fig 2B); alternatively, compression may be achieved indirectly by arranging for contraction of the muscle to inflate a cuff placed around the vessel ${ }^{10}$ (fig $2 \mathrm{C}$ ).

From one point of view, category 1 assists are the most conservative option, since the presence of an uninterrupted endothelium minimises the risk of thromboembolic complications. Small wonder, then, that cardiomyoplasty is the first, and so far the only, approach to have been used clinically.

\section{CATEGORY 2 ASSISTS}

These form part of an independent structure that is connected to the patient's circulation. As a result, an additional surface-which may be an artificial material or an autogenous biologica lining-is placed in contact with the blood. Again there are two main approaches:

(a) Skeletal muscle can be configured as an auxiliary pouch-shaped or tube-shaped ventricle by wrapping it around a suitable mandrel; 
the mandrel is subsequently removed, leaving a skeletal muscle ventricle (SMV) (fig 2D) or a skeletal muscle tube ventricle (SMT) (fig 2E) that can be connected to the circulation as a synchronous or counterpulsatory assist. ${ }^{411} 12$

(b) Skeletal muscle may be used to energise a mechanical artificial heart or ventricular assist device via a mechanical (fig $2 \mathrm{~F}$ ), hydraulic (fig $2 \mathrm{G}$ ), or electrical (fig $2 \mathrm{H}$ ) link. ${ }^{13} 14$

The fundamental limitation on the assists that can be obtained through these various approaches is the rate at which they allow energy to be converted to pumping work. We therefore consider first the power required for cardiac assistance. We go on to use recent experimental data from this laboratory to estimate the power available from skeletal muscle under ideal conditions. Finally we estimate the power that could be transferred into the circulation by the different configurations, and add comments on some of the practical difficulties.

\section{How much power is needed for cardiac assistance?}

The power required for pumping is related to the flow and the mean pressure by the expression:

\section{Power $=$ flow $\times$ pressure}

For a normal $70 \mathrm{~kg}$ subject with a body surface area of $1.7 \mathrm{~m}^{2}$ the mean flow at rest is about $6 \mathrm{l} / \mathrm{min} .{ }^{15}$ For an average systemic pressure of $100 \mathrm{~mm} \mathrm{Hg}$ this corresponds to about $1.3 \mathrm{~W}$. A capacity for at least $14 \mathrm{l} / \mathrm{min}$ should be built into the pump to support activities such as walking and climbing stairs, and it would therefore be appropriate to specify a short-term power capability of at least $3 \mathrm{~W}$. These levels are necessary where the patient's heart is in such poor condition that most of the systemic blood flow must be produced by the assist device. In less severe cases it may be acceptable to operate at lower levels, providing partial assistance that restores a reasonable quality of life while improving, or at least preventing further deterioration in, the condition of the patient's own heart.
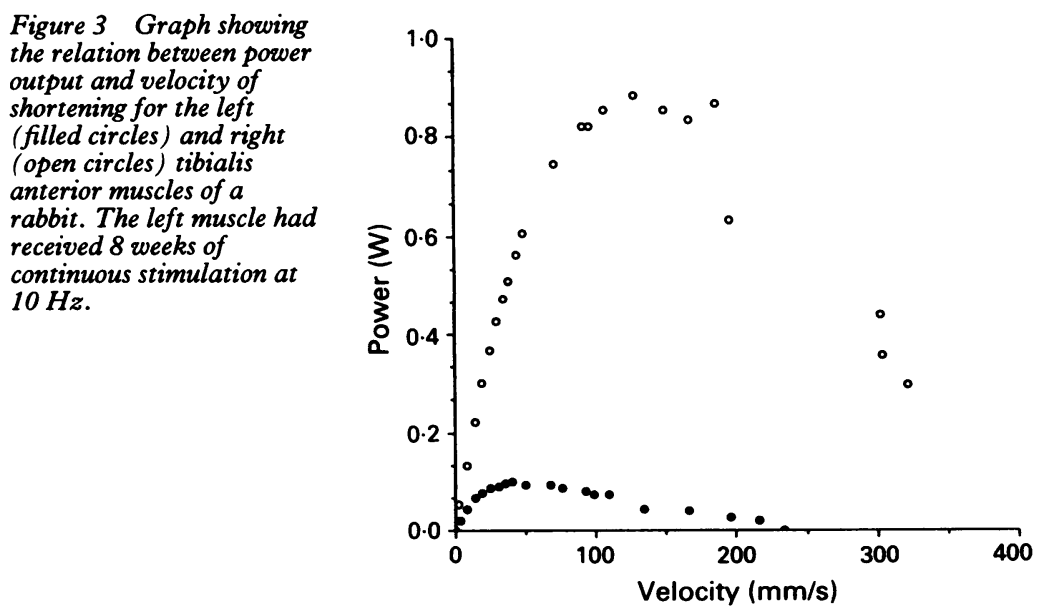

\section{How much power is available?}

No artificial energy source currently available can provide these levels of power from within the body. As a biological source, skeletal muscle has attracted interest because it allows a small expenditure of energy (needed to stimulate the motor nerve) to trigger the release of a potentially large amount of energy (derived ultimately from the normal intake of food and oxygen), which it converts with great efficiency into mechanical work.

The power available from skeletal muscle depends on two factors: the mass of wellvascularised tissue that can be recruited for the purpose and the sustainable power per gram of tissue. Whether one uses a dorsal muscle (such as latissimus dorsi) a ventral muscle (such as rectus abdominis) a muscle from the abdominal cavity (such as psoas major) or a muscle from the lower limb (such as gracilis or vastus lateralis), depends on the way in which power is to be coupled to the circulation. Some configurations require the muscle to be transposed into a new position, diverting it entirely from its normal function; other configurations would allow the muscle to operate in situ. The more indirect the linkage, the more freedom there is in the choice of a suitable muscle, but the poorer the efficiency of utilising the available energy.

For purposes of illustration, let us base our calculations on the generation of energy from a muscle mass of $600 \mathrm{~g}$, which happens to correspond to the average mass of an adult male latissimus dorsi muscle. We are seeking sustainable pumping power of $1.3 \mathrm{~W}$, with a reserve capacity that supports short-term use at up to at least $3 \mathrm{~W}$. The power required of a $600 \mathrm{~g}$ muscle would thus correspond to a sustainable $2 \cdot 1 \mathrm{~W} / \mathrm{kg}$ of muscle tissue, with the capability of working for shorter periods at $5 \mathrm{~W} / \mathrm{kg}$. To what extent is it feasible to expect these levels of work from skeletal muscle, bearing in mind that the human myocardium produces about $4 \mathrm{~W} / \mathrm{kg}$ at a resting rate?

To answer this question we have used an apparatus designed in our laboratory ${ }^{16}$ to characterise the mechanical performance of rabbit tibialis anterior (TA) muscles. From a power-velocity curve, such as that in fig 3 , we can obtain the rate of work output (force $x$ velocity) at any velocity of shortening from zero, where the force is maximal but the power is zero, to the maximum unloaded shortening velocity, where all the force produced is used to produce shortening within the muscle, and the external power output is again zero. Between these extremes there is a velocity for which the power output is maximal; this velocity is often referred to as $\mathrm{V}_{\mathrm{opr}}$. This curve describes the behaviour of a muscle only in single maximally activated contractions and does not, therefore, give any direct information about the endurance of the muscle. On the other hand, it shows very clearly that the power output of skeletal muscle is highly dependent on the load and that for a muscle to deliver its maximum power it must work against a load that allows it to shorten at a velocity close to $\mathrm{V}_{\mathrm{opr}}$.

For an unconditioned rabbit TA muscle, the 
maximum acute power output is about $300 \mathrm{~W} /$ $\mathrm{kg}$. After 8-10 weeks of continuous stimulation at a frequency of $10 \mathrm{~Hz}$, the mass falls to $50 \%$ of control and $\mathrm{V}_{\text {opt }}$ to $25 \%$ of control values. The combined effect of these losses is a reduction in power output to about $10 \%$ of control values $^{1718}$ (fig 3 , lower curve). This looks rather serious until it is recognised that in absolute terms the maximal acute power output of the stimulated muscle is still $30-40 \mathrm{~W} / \mathrm{kg}$, close to the systolic power of the left ventricular myocardium. These power outputs refer only to the rate at which work is performed during contraction. In any practical application, the muscle must spend at least as much time in relaxation as in contraction, and since work is available only during contraction the maximum power output is about $20 \mathrm{~W} / \mathrm{kg}$. At the specified work rates of $2 \cdot 1-5 \mathrm{~W} / \mathrm{kg}$, then, the stimulated muscle would be working at $10-20 \%$ of the maximum. To what extent can such working rates be sustained?

We have conducted fatigue tests on the same rabbit TA muscles under conditions in which they were constrained to perform external work at $10 \mathrm{~W} / \mathrm{kg}$. Conditioned muscles were able to maintain this level of work for many hours whereas control muscles showed progressive fatigue at the same initial work rate, even though their maximum power capacity was much greater. Although we have not run such tests at much higher rates of working, we doubt whether such rates could be sustained for more than short periods.

$10 \mathrm{~W} / \mathrm{kg}$ has to be regarded as an overestimate because it is based on rabbit muscle; the specific power of human muscle is intrinsically less, because of its slower contractile characteristics. ${ }^{19}$ The difference in power may be less marked when the comparison is made between human and rabbit muscles that are fully conditioned for continuous use, but it would seem sensible to allow for a reduction to $8 \mathrm{~W} / \mathrm{kg}$. The actual mass of muscle finally generating work will also be less than the starting mass, for several reasons. First, there is the loss of muscle bulk, already referred to, that is the consequence of performing work continuously at this high level; this stabilises at approximately $50 \%$ in the long term. ${ }^{6}$ This would reduce the working mass of a muscle such as latissimus dorsi to about $300 \mathrm{~g}$. Second, there is a loss associated with the grafting procedure. The experiments of Guerlinckx et $a^{20}$ on orthotopic grafting of rabbit rectus femoris muscles suggest that a chronic reduction in mass of $25 \%$ would be expected as a result of tendon section alone. This would bring about a further reduction to about $225 \mathrm{~g}$. To this must be added any loss of muscle fibres through ischaemic damage. The power available would therefore be unlikely to exceed $1.8 \mathrm{~W}$.

Recent work from our laboratory suggests that conditioning regimens can be devised that are very much better at preserving muscle mass and contractile speed, and hence the maximum power available in the long term. ${ }^{21} 22$ There is less scope for such techniques when the energy demand is continuously high, but they may be useful under partial assist conditions-for example, when only a proportion of the patient's heart beats are assisted.

\section{How may skeletal muscle power be harnessed?}

If the power requirements for cardiac assistance $(1.3-3 \mathrm{~W})$ are compared with the estimated maximum of $1.8 \mathrm{~W}$ available from skeletal muscle, it is immediately clear that, unless a substantially larger muscle mass could be recruited for the task, it is not feasible to attempt total replacement of more than resting heart function in this way. On the other hand, the estimated power available could, if appropriately harnessed, provide a very substantial assist (at an average systemic pressure of $100 \mathrm{~mm} \mathrm{Hg}, 1.8 \mathrm{~W}$ is equivalent to more than $8 \mathrm{l} / \mathrm{min}$ ). What is the best configuration for putting this power to use?

CONFIGURATIONS IN WHICH SKELETAL MUSCLE IS COUPLED DIRECTLY TO THE CIRCULATION Procedures based on reconfiguration of skeletal muscle alone have considerable advantages over any approach that requires the muscle to be interfaced with non-biological materials (see below). Of these procedures cardiomyoplasty and aortomyoplasty suffer from one serious limitation: the geometry of the pump is dictated by the size and shape of the existing organs. In some ways the consequences of this limitation are obvious: a grossly hypertrophied heart may be too large to be wrapped effectively by the patient's latissimus dorsi muscle; the small lumen of the aorta restricts the stroke volume that can be achieved by compressing it. But just as important as these anatomical considerations are the loading conditions that the fixed geometry imposes on the muscle wrap, because they constrain the muscle to operate far from the peak of its power curve.$^{1723}$ The benefits that have been reported by patients who have had the cardiomyoplasty procedure may be due to the reinforcement given to the myocardial wall, or to the translational motion imparted by contraction of the free portion of the graft. ${ }^{23}$

In contrast, the SMV approach is limited only by the size, shape, and fibre orientation of the latissimus dorsi (or other muscle) that is available. Subject only to these limitations, factors such as cavity volume, wall thickness, and direction of wrap are all within the control of the surgeon and can, given the appropriate basic knowledge, be optimised to provide the maximum pumping performance of which the muscle is capable. Set against this functional potential is the need to ensure that the introduction of such a device into the patient's circulation does not cause thrombus formation, with the attendant risks of obstruction of flow through the device and embolism to vital organs.

CONFIGURATIONS IN WHICH SKELETAL MUSCLE IS COUPLED INDIRECTLY TO THE CIRCULATION It is possible to envisage several ways in which a skeletal muscle could, in principle, provide the 
energy for a mechanical assist device, such as a left ventricular assist device (LVAD).

(a) Direct mechanical linkage (fig 2F) would be moderately efficient, especially if the muscle were allowed to operate in its normal line of action and without disturbance to its vascular supply. Problems would be the need for the assist device to be close to the muscle and the difficulty of creating a strong and reliable junction between a muscle and a non-biological surface. The latter is not a trivial matter: even tendon-to-tendon anastomoses take many months to acquire full strength. Furthermore the junction must be capable of unimpeded translational movement and must remain so.

(b) A hydraulic link could be established by wrapping a muscle around a fluid-filled bladder $^{24}$ (fig $2 \mathrm{G}$ ) or, more conservatively, by arranging for the bladder to divert the muscle from its normal line of action, so that it was compressed when the muscle contracted ${ }^{25}$ (fig $2 \mathrm{G})$. This approach would allow a little more freedom in the placement of the assist device relative to the muscle, at the cost of some resistive energy losses in the coupling lines. However, all existing biopolymers are permeable to some extent, so that in the present state of development it would not be possible to avoid a progressive escape of fluid from the system. This problem has yet to be overcome.

(c) An indirect approach (fig $2 \mathrm{H}$ ), in which contraction of the muscle was coupled to the generation of electricity, has several advantages. It is the most flexible arrangement in terms of placement: for example, an intrathoracic device might be powered by a muscle in the lower limb. There would be a broader choice of assist device, and this could encompass electrically driven devices that had not been designed with skeletal muscle power specifically in mind. However, such an approach poses technological problems that await a reliable solution, and these include the problems of muscle attachment already mentioned.

With an optimistic estimate of $50 \%$ efficiency for the energy conversions involved, the maximum pumping power available from human muscle working at $8 \mathrm{~W} / \mathrm{kg}$ would be $0.9 \mathrm{~W}$, equivalent to $4 \mathrm{l} / \mathrm{min}$ for the mechanical and hydraulic approaches. The electromechanical approach involves two conversions, and the maximum pumping power available would therefore be $0.45 \mathrm{~W}$, equivalent to $2 \mathrm{l} / \mathrm{min}$. Thus partial assistance of resting heart function is bordering on feasibility with this technique, but the reserve capacity would be limited.

One of the attractions of this general approach is that it would capitalise on the results of an extensive research effort over several years. For example, devices are already available in which the flow has been well characterised and clinical experience has been accumulated. A major advantage of mechanical devices is that they can provide cardiac assistance as soon as they have been connected to the patient's circulation. Thus in the immediate postoperative period it might be possible to energise the device from an external power source, transferring to endogenous power from muscle after the conditioning process had been completed.

A less obvious advantage of using skeletal muscle as the power source for another device is that it eliminates problems of synchrony with the cardiac cycle. We have already stated that during extreme conditioning the shortening velocity for maximum power, $\mathrm{V}_{\mathrm{opt}}$, expressed in fibre lengths per second, declines to about $25 \%$ of control values. Apart from the associated power loss, which has already been referred to, the slowing of contraction and relaxation limits the amount of shortening that can take place within one or other phase of the cardiac cycle. ${ }^{23}$ Even if the load were optimised to allow the muscle to shorten at $\mathrm{V}_{\mathrm{opt}}$, a shortening of $20 \%$ would take $400 \mathrm{~ms}$ at 0.5 fibre lengths/s. Such considerations become irrelevant if the muscle can be allowed to operate asynchronously.

A major disadvantage of mechanical assist devices is their high cost. This would be increased still further in the case of a device powered by skeletal muscle, which would also require an implantable energy converter and a pacemaker-like neuromuscular stimulator. The long-term success of mechanical circulatory devices is still limited by the biomaterials used in their construction. Materials in current use have a useful life of only about 18 months, but progress in this area has been rapid and this figure could be expected to improve. Reference has already been made to the problems of anchoring the muscle to an artificial device, and the additional problems of leakage associated with a hydraulic system. It is not a trivial anatomical problem to accommodate a mechanical device in the chest when the patient's own heart is still in situ. There is also the problem of finding room for the power converter, although this would be less serious in the case of the electrically driven assist. Finally, all of the existing clinical experience with mechanical circulatory devices has been gained in bridge-to-transplant procedures, and it would be necessary to develop surgical techniques that were not likely to compromise the action of the patient's own heart if such devices were to be used to provide a permanent assist.

\section{Conclusions}

When total heart replacement is called for and a transplant is unsuitable or unavailable the mechanical artificial heart is the only option. Mobility, without restraint or encumbrance, should be a major goal of cardiac rehabilitation, so it is appropriate to consider the prospects for implantable devices that are independent of exogenous power sources. We show here that there is little hope of obtaining the energy for such a device entirely from the patient's own skeletal muscle: an electromechanical pump energised in this way would be limited to about $2 \mathrm{l} / \mathrm{min}$, with little in reserve. This is an unsatisfactory outcome for a procedure that is likely to be very expensive. The picture could change if highly efficient energy converters could be harnessed to a much larger muscle 
mass, but in the present state of development the outlook for this approach is not encouraging. Direct mechanical or hydraulic coupling is potentially more efficient, but requires the assist device to be placed fairly close to the energising muscle. Mechanical and electromechanical approaches pose problems of muscle attachment and the hydraulic approach poses problems of fluid leakage.

Much more power is available if the muscle tissue can be employed directly. With the muscle formed into a pumping chamber and loaded so as to operate around the peak of the power curve, there is sufficient power to produce an average flow as high as $81 / \mathrm{min}$. Such an arrangement need not involve mechanical pumps or energy convertors and the hardware cost would therefore be limited to that of an implantable neuromuscular stimulator and, possibly, a valve. The considerable progress that has been made with this type of approach has been the subject of recent reviews. ${ }^{326}$ Although such configurations could in theory replace the resting function of the left ventricle, a more practical objective would be a partial assist of $1-21 / \mathrm{min}$, with the muscle working for much of the time below its maximum capacity. Under these conditions, there would be a better prospect of preserving the contractile speed of the conditioned muscle, with corresponding benefits for the amplitude and duration of each assist cycle and a substantial improvement in the reserve pumping capacity. This, then, is an avenue that could lead to major advances in the surgical treatment of chronic cardiac failure.

We thank the British Heart Foundation and the Science and Engineering Research Council for their support of this work.

1 Salmons S, Henriksson J. The adaptive response of skeletal muscle to increased use Musc Nerve 1981;4:94-105.

2 Pette $D$, Vrbová $G$. Neural control of phenotypic expression in mammalian muscle fibers. Musc Nerve 1985;8:676-89.

3 Salmons S, Jarvis JC, eds. Harnessing skeletal muscle power for cardiac assistance. Commission of the European Communities, 1991;ISBN 90-365-0412-0, 123pp.

4 Acker MA, Hammond R, Mannion JD, Salmons $S$ Stephenson $\mathrm{LW}$. An autologous biologic pump motor $J$ Thorac Cardiovasc Surg 1986;12:733-46.

5 Clark BJ III, Acker MA, McCully K, Subramanian HV Hammond RL, Salmons $S$, et al. In vivo ${ }^{31} \mathrm{P}-\mathrm{NMR}$ spectroscopy of chronically stimulated canine skeletal muscle. Am J Physiol 1988;254:C258-66.

6 Mayne C, Anderson WA, Hammond RL, Eisenberg BR, Stephenson LW, Salmons S. Correlates of fatigue resistance in canine skeletal muscle stimulated electrically for up to one year. Am J Physiol: Cell Physiol 1991;261 (Cell Physiol 30):C259-70.

7 Chagas ACP, Moreira LFP, da Luz PL, Camarano GP Leirner A, Stolf NAG, et al. Stimulated preconditioned skeletal muscle cardiomyoplasty. An effective means of cardiac assist. Circulation 1989;80 (Suppl III):202-8.

8 Hagege AA, Desnos M, Chachques J-C, Carpentier A, Fernandez F, Fontaliran F, et al. Preliminary report: follow-up after dynamic cardiomyoplasty. Lancet 1990; 335:1122-4.

9 Pattison CW, Cumming DVE, Williamson A, ClaytonJones DG, Dunn MJ, Goldspink G, Yacoub M. Aortic counterpulsation for up to 28 days with autologous counterpulsation for up to 28 days with autologous latissimus dor

10 Kochamba G, Desrosiers C, Dewar M, Chiu R-C. The muscle-powered dual-chamber counterpulsator: rheoogically superior implantable cardiac assist device. Ann Thorac Surg 1988;45:620-5.

11 Anderson DR, Pochettino A, Hammond RL, Hohenhause $\mathrm{E}$, Spanta AD, Bridges CR Jr, et al. Autogenously lined skeletal muscle ventricles in circulation. Up to nine months' experience. J Thorac Cardiovasc Surg 1991; 101:661-70.

12 Pochettino A, Anderson DR, Hammond RL, Salmons S, Stephenson LW. Skeletal muscle ventricles. Seminars Thorac Cardiovasc Surg 1991;3:154-9.

13 Frey $M$. Thoma $H$, Gruber $H$, Stöhr $H$, Havel $M$. The chronically stimulated psoas muscle as an energy source for artificial organs: an experimental study in sheep. In for artificial organs: an experimental study in sheep. In Chiu RC-J, ed. Biomechanical cardiac assist. Cardiomyoplasty and muscle-powered devices. Mount Kisco,

14 Ugolini F. Skeletal muscle for artificial heart drive: theory and in vivo experiments. In: Chiu RC-J, ed. Biomechanical cardiac assist. Cardiomyoplasty and muscle-powered devices. Mount Kisco, New York: Futura Publishing Company, 1986:193-210

15 Hurst JW, ed. The heart, arteries and veins. New York: McGraw-Hill Book Company, 4th ed, 1978:76.

16 Jarvis JC, Salmons S. An electrohydraulic apparatus for the measurement of static and dynamic properties of rabbit measurement of static and dynamic prope

17 Salmons S, Jarvis JC. Cardiomyoplasty: the basic issues. Cardiac Chron 1990;4:1-7.

18 Salmons S, Jarvis JC. The working capacity of skeletal muscle transformed for use in a cardiac assist role. In: muscle transformed for use in a cardiac assist role. In:
Chiu RC-J, Bourgeois IM, eds. Transformed Muscle for Chiu RC-J, Bourgeois IM, eds. Transformed Muscle for
Cardiac Assist and Repair. Mount Kisco, NY: Futura Cardiac Assist and Repair. Mount
Publishing Company, 1990:89-104.

19 Faulkner JA, Claflin DR, Brooks SV, Burton HW. Powe output of fiber segments from human latissimus dor muscles: implications for heart assist devices. In: Carraro U, Salmons S, eds. Basic and Applied Myology: Perspectives for the 90's. Padua: Unipress, 1992:31-8.

20 Guerlinckx PJ, Faulkner JA, Essig DA. Neurovascularanastomosed muscle grafts in rabbits: functional deficits result from tendon repair. Musc Nerve 1988;11:745-51.

21 Jarvis JC, Mayne CN, Little P, Sutherland H, Brownson C, Salmons S. Use of $2.5 \mathrm{~Hz}$ and $10 \mathrm{~Hz}$ stimulation to examine coordination in the phenotypic response of fast examine coordination in the phenotypic response of fast 1991;13:237.

22 Jarvis JC, Mayne CN, Salmons S. Basic studies on skeletal muscle for cardiac assistance. J Cardiac Surg 1991;6 muscle for cardi

23 Salmons S, Jarvis JC. Cardiomyoplasty: a look at the fundamentals. In: Carpentier A, Chachques J-C, Grandjean P, eds. Cardiomyoplasty. Mount Kisco, NY: Futura Publishing Company, 1991:3-17.

24 Anderson WA, Bridges CR, Chin AJ, Andersen JS, Acke MA, Hammond RL, et al. Long-term neurostimulation of skeletal muscle: its potential for a tether-free biologic cardiac assist device. $P A C E$ 1988;11:2128-34.

$25 \mathrm{Li} \mathrm{CM}$, Hill A, Colson M, Desrosiers C, Chiu RC-J. Implantable rate-responsive counterpulsation assist system. Ann Thorac Surg 1990;49:356-62.

26 Hooper TL, Stephenson LW. Using skeletal muscle to assist the heart. Br Heart J 1991;66:261-3. 